\title{
Shedding a Little Light on the Need for Scheduled ICU Admission Following Endovascular Treatment of Unruptured Intracranial Aneurysm
}

\author{
Craig A. Williamson ${ }^{1,2^{*}}$
}

@ 2021 Springer Science+Business Media, LLC, part of Springer Nature and Neurocritical Care Society

By their nature, many neurological emergencies, particularly cerebrovascular emergencies, arise suddenly and without warning, typically requiring rapid diagnosis and intervention to have any chance to prevent permanent injury to life or limb. Despite numerous advances in neurological monitoring, the most reliable and effective tool to identify actual or impending neurological emergencies remains serial examinations performed by trained personnel. The United States Center for Medicare and Medicaid Services defines critical illness as one that "Acutely impairs one or more vital organ systems such that there is a high probability of imminent or life threatening deterioration in the patient's condition" [1]. Because of the actual or perceived high risk of developing a delayed but acute, intervenable complication, patients have traditionally been monitored in an intensive care unit (ICU) following most elective intracranial procedures, including endovascular aneurysm treatment.

Scheduled ICU admission following intracranial procedures has intuitive benefits, particularly the availability of trained nursing staff who can perform frequent neurological and vital signs monitoring combined with the presence of medical providers who are capable of intervening if an emergent complication does occur. At the same time, as with all medical interventions, ICU monitoring

\footnotetext{
*Correspondence: craigaw@med.umich.edu

${ }^{1}$ Department of Neurosurgery, University of Michigan, 1500 E. Medical Center Drive, Ann Arbor, MI 48109, USA

Full list of author information is available at the end of the article

This comment refers to the article available online at https://doi.org/10. 1007/s12028-021-01306-0.
}

also carries risks. Continuously performing hourly neurological examinations overnight following aneurysm coiling increases the likelihood that a delayed complication will be identified earlier, but also can reasonably be expected to increased complications secondary to invasive monitoring devices, poor sleep, and delirium. In addition, ICU admission has real financial and opportunity costs [2]. The United States has more ICU beds per capita than many other developed countries, but the fact that ICU beds and trained ICU nurses are a valuable and finite resource was made explicit by the recent coronavirus disease 2019 pandemic [3, 4]. Furthermore, clinical experience and recent research suggests that as operative experience, devices, and techniques continuously improve, there is a corresponding decrease in complication rates following elective procedures, implying that the equilibrium point in which the benefits of ICU admission is balanced by the risks and costs is constantly shifting [5].

In this context, several recent publications have attempted to quantify the frequency with which ICUspecific interventions are required following elective neurosurgical procedures, identify risk factors for requiring ICU level of care, or determine potential cost savings associated with receipt of care outside of the ICU [6-10]. Importantly, these studies draw a clear distinction between intensive monitoring via frequent neurological assessments, which are generally performed by trained nurses and can increasingly be provided outside of a traditional ICU in intermediate care units, and intensive care that typically requires active physician involvement and decision-making. In this issue, Padmanaban et al. [11] 
add to this growing body of literature by investigating the frequency and risk factors for ICU-specific care in a large cohort of patients following endovascular treatment of unruptured intracranial aneurysms at a single academic medical center. The authors provide a reasonable and rigorous definition of ICU-specific care, a necessary, somewhat nebulous concept that will inevitably vary between health systems and institutions. Based on their definition, ICU needs were only present following $13.6 \%$ of procedures. More importantly, $75 \%$ of the time an ICU need was clearly present at the start of ICU admission, and most other ICU-specific needs developed relatively early. The onset of delayed neurological deficits $(>10 \mathrm{~h}$ following ICU admission) occurred in less than $1 \%$ of cases. A small number of common-sense risk factors (age, procedural complications, and procedural length) were significantly associated with increasing odds of ICU need.

The limitations of this study are obvious and appropriately acknowledged by the authors. Significant caution should be exercised when generalizing these results from a single-center study to other institutions, particularly settings with varying resources, procedural volumes, and systems of care. At the same time, this and other similar studies provide support to centers that wish to experiment with alternative care models for certain elective intracranial procedures outside of traditional ICUs. Beyond evaluating effects on patient outcomes and financial costs, future studies should examine indirect costs and benefits, such as hospital length of stay and complications, changes in neurological ICU bed availability, emergency department boarding times for patients with neurological emergences, postanesthesia care unit wait times for patients with neurosurgery needs, and the timely acceptance of outside hospital transfers for patients with neurological emergencies.

It is also important to identify the optimal dose of ICU care and neuromonitoring for individual patients undergoing elective procedures in terms of length of stay and frequency of neurological assessments. At present, decisions on both are largely guided by custom and opinion rather than any objective data, making them fertile ground for comparative effectiveness studies. Regardless of where they are cared for, patients with neurological diseases will sometimes experience rapid and unexpected deterioration, so appropriate policies and personnel who can quickly stabilize and transfer patients to an appropriate level of care must be insured. Proceduralists and intensivists will always need to work collaboratively to determine the optimal setting for patients who are postoperative, being mindful of their local resources and ready to adapt to changing bed availability and manage the inevitable unexpected complications. But it would certainly be nice to not make these decisions in a data vacuum, so hopefully this study will spur others to rigorously investigate optimal systems of care for patients following endovascular and other elective intracranial procedures.

\section{Author details \\ ${ }^{1}$ Department of Neurosurgery, University of Michigan, 1500 E. Medical Center Drive, Ann Arbor, Ml 48109, USA. ${ }^{2}$ Department of Neurology, University of Michigan, Ann Arbor, MI, USA.}

\section{Source of Support}

There was no external funding for this article.

\section{Conflicts of Interest}

The author reports the following conflict of interest disclosures: research grant support from the United States Department of Defense and Xoran, Inc; and Honoraria from UpToDate Inc, the Massachusetts Medical Society, and Oakstone Publishing.

\section{Publisher's Note}

Springer Nature remains neutral with regard to jurisdictional claims in published maps and institutional affiliations.

Received: 9 July 2021 Accepted: 12 July 2021

Published online: 30 July 2021

\section{References}

1. Department of Health and Human Services; Centers for Medicare and Medicaid Services. CMS Manual System: Pub 100-04 Medicare Claims Processing Transmittal 2997. 2014. https://www.cms.gov/Regulationsand-Guidance/Guidance/Transmittals/downloads/R2997CP.pdf. Accessed 7 March 2021.

2. Ghaffar S, Pearse RM, Gillies MA. ICU admission after surgery: Who benefits? Curr Opin Crit Care. 2017;23(5):424-9.

3. Wunsch $H$, Angus DC, Harrison DA, et al. Variation in critical care services across North America and Western Europe. Crit Care Med. 2008;36(10):2787-93, e1-8.

4. Nurok M, Kahn J. Intensive care unit capacity, cancellation of elective surgery, and the US pandemic response. Anesth Analg. 2020;131(5):1334-6.

5. Cote DJ, Karhade AV, Larsen AMG, et al. United States neurosurgery annual case type and complication trends between 2006 and 2013: an American College of Surgeons National Surgical Quality Improvement Program Analysis. J Clin Neurosci. 2016;31:106-11.

6. Burrows AM, Rabinstein AA, Cloft $\mathrm{HJ}$, et al. Are routine intensive care admissions needed after endovascular treatment of unruptured aneurysms? Am J Neuroradiol. 2013;34:2199-201.

7. Gabel BC, Martin J, Crawford JR, et al. Questioning the need for ICU level of care in pediatric patients following elective uncomplicated craniotomy for brain tumors. J Neurosurg Pediatr. 2016;17:564-8.

8. Hanak BW, Walcott BP, Nahed BV, et al. Postoperative intensive care unit requirements after elective craniotomy. World Neurosurg. 2014:81:165-72.

9. Franko LR, Hollon T, Linzey J, et al. Clinical factors associated with ICUspecific care following supratentoral brain tumor resection and validation of a risk prediction score. Crit Care Med. 2018;46:1302-8.

10. Stetler WR, Griauzde J, Saadeh Y, et al. Is intensive care monitoring necessary after coil embolization of unruptured intracranial aneurysms? J Neurointerv Surg. 2017:9:756-60.

11. Padmanaban V, Gigliotti M, Majid S, et al. Risk factors associated with ICU-specific care in patients undergoing endovascular treatment of unruptured intracranial aneurysms. Neurocrit Care. 2021. https://doi.org/ 10.1007/s12028-021-01306-0. 